\title{
Abnormal inhibition of osteoclastogenesis by mesenchymal stem cells through the miR-4284/CXCL5 axis in ankylosing spondylitis
}

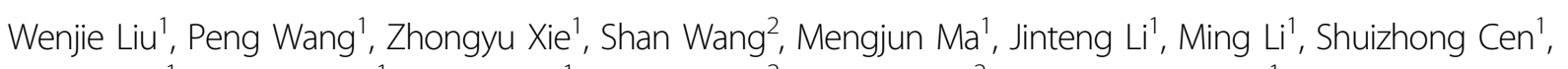
Su'an Tang', Guan Zheng ${ }^{1}$, Guiwen $\mathrm{Ye}^{1}$, Xiaohua $\mathrm{Wu}^{2}$, Yanfeng $\mathrm{Wu}^{2}$ and Huiyong Shen ${ }^{1}$

\begin{abstract}
Ankylosing spondylitis (AS) is a common inflammatory autoimmune disease, characterized by pathological osteogenesis. Mesenchymal stem cells (MSCs), as the main source of osteoblasts, participate in bone remodeling not only through differentiation into osteoblasts but also through indirect regulation of osteoclastogenesis. Our previous study indicated that the stronger osteogenic differentiation of MSCs from AS patients (ASMSCs) involved in pathological osteogenesis. However, whether there is any abnormality in the regulation of osteoclastogenesis by ASMSCs remains unclear. In this study, ASMSCs or MSCs from healthy donors (HDMSCs) were co-cultured with CD14 + monocytes in osteoclast induction medium. Our results demonstrated that ASMSCs exhibited a stronger capacity to inhibit osteoclastogenesis than HDMSCs. To explore underlying mechanisms, cytokine array assays were performed, showing that ASMSCs secreted more CXCL5 than HDMSCs, which was confirmed by enzyme-linked immunosorbent assays. Moreover, inhibition of osteoclastogenesis by ASMSCs was recovered by decreasing CXCL5. Besides, the inhibitory effect of CXCL5 on osteoclastogenesis was confirmed by exogenous addition. Bioinformatics analysis was applied to find the interaction between miR-4284 and CXCL5, which was verified by luciferase reporter assays. Furthermore, we used miR-4284 inhibitors or mimics to prove that the expression of CXCL5 was regulated by miR-4284. Further analysis showed that downregulation of miR-4284 in MSCs resulted in increase of CXCL5, markedly inhibiting osteoclastogenesis, whereas upregulation of miR-4284 in MSCs had the opposite effect. Our findings indicate that ASMSCs exhibit a stronger capacity to inhibit osteoclastogenesis than HDMSCs through the miR-4284/ CXCL5 axis, which provide a new perspective on the mechanism of pathologic osteogenesis in AS.
\end{abstract}

\section{Introduction}

Ankylosing spondylitis (AS) is a common inflammatory autoimmune disease that mainly affects the axial skele$\operatorname{ton}^{1}$. Although the pathogenesis of AS remains unknown,

\footnotetext{
Correspondence: Yanfeng Wu (wuyf@mail.sysu.edu.cn) or

Huiyong Shen (shenhuiy@mail.sysu.edu.cn)

'Department of Orthopedics, Sun Yat-sen Memorial Hospital, Sun Yat-sen

University, Guangzhou 510120, People's Republic of China

${ }^{2}$ Center for Biotherapy, Sun Yat-sen Memorial Hospital, Sun Yat-sen University,

Guangzhou 510120, People's Republic of China

These authors contributed equally: Wenjie Liu, Peng Wang, Zhongyu Xie

Edited by I. Amelio
}

genetic, environmental, and immunological factors may be involved ${ }^{2,3}$. Pathological osteogenesis is one of the central features of $\mathrm{AS}^{4}$, though the mechanism is still unclear. Early diagnosis of pathological osteogenesis is currently difficult, and successful therapy for pathological osteogenesis has not yet been defined ${ }^{5}$. As many patients ultimately develop spinal ankylosis or hip joint ankylosis, which seriously affects quality of life ${ }^{4}$, it is important to study the pathogenesis of the osteogenesis that occurs in AS and to explore effective methods for early diagnosis and treatment.

\section{(c) The Author(s) 2019}

(c) (i) Open Access This article is licensed under a Creative Commons Attribution 4.0 International License, which permits use, sharing, adaptation, distribution and reproduction in any medium or format, as long as you give appropriate credit to the original author(s) and the source, provide a link to the Creative Commons license, and indicate if changes were made. The images or other third party material in this article are included in the article's Creative Commons license, unless indicated otherwise in a credit line to the material. If material is not included in the article's Creative Commons license and your intended use is not permitted by statutory regulation or exceeds the permitted use, you will need to obtain permission directly from the copyright holder. To view a copy of this license, visit http://creativecommons.org/licenses/by/4.0/. 
Mesenchymal stem cells (MSCs) are the main source of osteoblasts ${ }^{6}$. We previously found that MSCs from AS patients (ASMSCs) displayed a stronger capacity to differentiate into osteoblasts than MSCs from healthy donors (HDMSCs), indicating the involvement of ASMSCs in pathological osteogenesis ${ }^{7}$. Osteoblasts and osteoclasts are the two key types of cells in bone remodeling ${ }^{8-10}$; MSCs participate in bone remodeling not only through differentiation into osteoblasts but also through indirect regulation of osteoclastogenesis ${ }^{11,12}$. However, whether there is any abnormality in the regulation of osteoclastogenesis by ASMSCs remains unclear.

Human osteoclasts are largely derived from $\mathrm{CD} 14^{+}$ monocytes ${ }^{13}$, and stimulation with monocyte colonystimulating factor (M-CSF) and receptor activator of NF- $\mathrm{BB}$ ligand (RANKL) can cause monocytes to differentiate into osteoclasts ${ }^{14}$. Nuclear factor of activated T cells c1 (NFATc1) is the master transcription factor for osteoclastogenesis ${ }^{14}$. The major function of osteoclasts is bone resorption, which primarily depends on tartrate-resistant acid phosphatase (TRAP) and cathepsin K (CTSK $)^{15}$. Many inflammatory cytokines regulate osteoclastogenesis ${ }^{16}$. For example, IL-4 and IL-10 inhibit osteoclastogenesis, whereas SDF-1 and MCP-1 stimulate osteoclastogenesis ${ }^{16-18}$. Previous studies have shown that CXCL5 has a strong effect on neutrophil recruitment and is involved in a variety of inflammatory diseases, such as rheumatoid arthritis (RA) and pediatric ulcerative colitis ${ }^{19-21}$, yet its role in AS remains unknown.

MicroRNAs (miRNAs) are small noncoding RNAs that regulate gene expression by causing mRNA degradation or translational inhibition ${ }^{22}$ through interaction via a seed sequence (2-7 nucleotide) in the 3'-untranslated region of the target $\mathrm{mRNA}^{22}$. miRNAs play an important role in many processes, including cell proliferation, differentiation, and apoptosis ${ }^{16}$. Abnormal expression of miRNAs is involved in the development of many autoimmune diseases, such as RA and systemic lupus erythematosus ${ }^{23}$. For example, compared to healthy donors (HDs), higher levels of miR-146a, miR-125a, miR-151a, and miR-22 and lower levels of miR-150 and miR-451a have been found in AS patients, and these miRNAs can serve as biomarkers of disease activity in $\mathrm{AS}^{24}$. These results suggest that miRNAs may participate in the pathology of AS.

In this study, we investigated the effect of ASMSCs compared with HDMSCs on osteoclastogenesis and explored the mechanism of abnormal inhibition of osteoclastogenesis by MSCs in AS. Our results demonstrate that the ability to inhibit osteoclastogenesis is enhanced in ASMSCs compared with HDMSCs through secretion of CXCL5, which can be regulated by miR-4284. These findings provide a new perspective on the mechanism of pathologic osteogenesis in AS.

\section{Materials and methods}

\section{Cell isolation and culture}

In this study, $30 \mathrm{HDs}$ and 30 AS patients who satisfied the modified New York criteria ${ }^{25}$ were recruited. Details of the study subjects were showed in Supplementary Table S1. The protocol was approved by the Ethics Committee of Sun Yat-sen Memorial Hospital (Sun Yat-sen University, Guangzhou, China), and informed consent was obtained from all subjects. MSCs were isolated and purified as previously described ${ }^{7}$ and cultured in Dulbecco's modified Eagle's medium (Gibco) containing 10\% fetal bovine serum (Sijiqing Biological Engineering Material Company). The culture medium was replaced every 3 days.

Whole blood was obtained from HDs with the permission of the Ethics Committee of Sun Yat-sen Memorial Hospital. Peripheral blood mononuclear cells (PBMCs) were isolated from whole blood using density-gradient centrifugation as previously described ${ }^{26}$. CD1 $14^{+}$monocytes were isolated via positive selection of PBMCs labeled with magnetic-beadconjugated anti-human CD14 mAbs according to the protocol provided by the manufacturer (Miltenyi Biotech). For assessment of $\mathrm{CD} 14^{+}$monocyte purity, cells were incubated with fluorescein isothiocyanate (FITC)-conjugated anti$\mathrm{CD} 14 \mathrm{mAb}$ (BD Biosciences) on ice for $30 \mathrm{~min}$, washed three times with phosphate-buffered saline (PBS) and detected using a BD Influx cell sorter (BD Biosciences). All cells were cultured at $37^{\circ} \mathrm{C}$ in $5 \% \mathrm{CO}_{2}$.

\section{Co-culture system for osteoclast differentiation}

CD14 $4^{+}$monocytes $\left(2.5 \times 10^{5} / \mathrm{cm}^{2}\right)$ were seeded in the lower wells and MSCs (monocytes: MSCs $=10: 1$ ) in the upper wells of transwell plates $(0.4 \mu \mathrm{m}$ pore size; Corning). For induction of osteoclast differentiation, $\mathrm{CD} 14^{+}$monocytes $\left(2.5 \times 10^{5} / \mathrm{cm}^{2}\right)$ were cultured in $\alpha$-minimum essential medium containing $10 \%$ fetal bovine serum supplemented with $25 \mathrm{ng} / \mathrm{mL}$ recombinant human M-CSF and $50 \mathrm{ng} / \mathrm{mL}$ recombinant human RANKL (both from Peprotech). The medium was replaced every 3 days.

\section{TRAP staining}

Cells were washed three times with PBS on day 9 and stained for TRAP activity using the leukocyte acid phosphatase kit (Sigma) according to the manufacturer's instructions. TRAP-positive cells with at least three nuclei were considered osteoclasts. At least nine fields of view covering the entire plate were assessed, and the mean number of osteoclasts was calculated.

\section{F-actin assay}

Cells were fixed for 5 min with 4\% paraformaldehyde on day 9 and washed extensively with PBS. The cells were then stained with FITC-conjugated phalloidin (Sigma) and 4', 6-diamidino-2-phenylindole for $40 \mathrm{~min}$ at room temperature. Afterwards, the cells were washed three 
times with PBS and observed with an Axio Observer fluorescence microscope (Carl Zeiss).

\section{Bone resorption assay}

For determination of the capacity of osteoclasts to resorb bone, CD14 $4^{+}$monocytes $\left(2.5 \times 10^{5} / \mathrm{cm}^{2}\right)$ were plated onto bovine cortical slices in 24-well plates and cultured with MSCs in osteoclast differentiation medium for up to 15 days. To evaluate bone resorption, the slices were washed thoroughly with PBS and fixed with $2.5 \%$ glutaraldehyde for $15 \mathrm{~min}$. The slices were then washed three times with PBS and treated with ultrasonication in $0.5 \mathrm{M}$ $\mathrm{NH}_{4} \mathrm{OH}$ to remove adherent cells. After washing three times with PBS, the slices were gradient-dehydrated in 70 , 95 , and $100 \%$ ethanol, stained for 5 min with $1 \%(\mathrm{w} / \mathrm{v})$ toluidine blue and washed five times with PBS. At least nine fields of view covering the entire slice were assessed, and the mean number of pit formations was calculated.

\section{Real-time quantitative reverse transcription-polymerase chain reaction ( $q R T-P C R$ )}

Cells were washed three times with PBS, and total RNA was extracted after the addition of TRIzol (Invitrogen). Complementary DNA was transcribed from $1 \mu \mathrm{g}$ of RNA using a PrimeScript RT reagent kit (TaKaRa) according to the instructions. qRT-PCR was carried out using a LightCycler ${ }^{\circledR} 480$ PCR system (Roche) with SYBR Premix Ex Taq (TaKaRa). The steps were described in our previously published research ${ }^{27}$. The procedure for the qRT-PCR assay was $95^{\circ} \mathrm{C}$ for $30 \mathrm{~s}$ and 40 cycles at $95^{\circ} \mathrm{C}$ for $5 \mathrm{~s}$ and $60^{\circ} \mathrm{C}$ for $20 \mathrm{~s}$. Each sample was performed in triplicate, and the mean mRNA level was calculated. To confirm specific amplification of targets, the melting curve for each sample was analyzed. GAPDH or U6 was used as internal control, and the relative expression of each gene was calculated using the $2^{-\Delta \Delta C t}$ method. The forward and reverse primers used for target genes are available in Supplementary Table S2.

\section{Western blot analysis}

Cells were lysed in RIPA buffer (Sigma) supplemented with $1 \%$ protease and phosphatase inhibitors (Roche) for $30 \mathrm{~min}$ on ice. The lysates were then centrifuged at $12,000 \mathrm{rpm}$ for $30 \mathrm{~min}$ at $4{ }^{\circ} \mathrm{C}$, and the proteins in the supernatant were quantified using a BCA assay kit (Sigma) according to the manufacturer's instructions. Equal amounts of protein diluted in sodium dodecyl sulfate (SDS) loading buffer, separated by $10 \%$ SDS-polyacrylamide gel electrophoresis, and electrotransferred onto polyvinylidene fluoride membranes (EMD Millipore Billerica). The membranes were blocked with $5 \%$ skim milk in TBST at room temperature for $60 \mathrm{~min}$ and incubated overnight at $4{ }^{\circ} \mathrm{C}$ with primary antibodies against GAPDH, TRAP, CTSK, NFATc1, p65, phosphorylated p65, phosphorylated p38, p38, ERK-1/2, phosphorylated ERK-1/2, JNK, and phosphorylated JNK (each diluted
1:1000; Cell Signaling Technology). Afterwards, the membranes were washed three times with TBST and incubated with appropriate horseradish peroxidase (HRP)-conjugated secondary antibodies (diluted 1:3000; Santa Cruz Biotechnology) at room temperature for $60 \mathrm{~min}$. The membranes were washed three times with TBST, and signals were detected using Immobilon Western Chemiluminescent HRP Substrate (Millipore).

\section{Cytokine array assay}

MSCs $\left(2.5 \times 10^{4} / \mathrm{cm}^{2}\right)$ were seeded into 12 -plate wells. After 3 days, culture supernatants were collected from HDMSCs and ASMSCs, respectively. Then culture supernatants of MSCs were analyzed using Proteome Profiler Human XL Cytokine Array Kit (R\&D Systems) according to the manufacturer's instructions. For each assay, $500 \mu \mathrm{L}$ of cell culture supernatant was used, and quantification of cytokine optical densities was obtained with HLImage ++ software (Western Vision). Densitometry of signal intensities was performed to quantify differences in cytokines between ASMSCs and HDMSCs.

\section{Enzyme-linked immunosorbent assay (ELISA)}

MSCs $\left(2.5 \times 10^{4} / \mathrm{cm}^{2}\right)$ were seeded into 12-plate wells and supernatants of MSCs were harvested on day 3. The level of CXCL5 protein in the cell culture supernatant was quantitated using Human CXCL5 Quantikine ELISA Kit (R\&D Systems) following the manufacturer's protocol.

\section{Lentivirus construction and infection}

A lentivirus encoding a short hairpin RNA (shRNA, GenePharma) for CXCL5 (sh-CXCL5) was constructed; the target sequence was 5'-AGAGCUGCGUUGCGUU UGUTT- $3^{\prime}$. The sequence for the negative control was 5'-UUCUCCGAACGUGUCACGUTT-3'. For transfection, the lentivirus and polybrene $(5 \mu \mathrm{g} / \mathrm{mL})$ were added to the MSC culture medium for $24 \mathrm{~h}(\mathrm{MOI}=50)$.

\section{Exogenous CXCL5 assay}

Recombinant human CXCL5 (R\&D Systems) was added to the culture medium at different concentrations $(1,5$, 25 , and $125 \mathrm{ng} / \mathrm{mL}$ ) every 3 days. Cells were grown in the presence of M-CSF $(25 \mathrm{ng} / \mathrm{mL})$ and RANKL $(50 \mathrm{ng} / \mathrm{mL})$. After 12 days, the cells were stained for TRAP and F-actin, and after 15 days, resorption pits on the slices were stained with toluidine blue.

\section{Luciferase reporter assay}

A CXCL5 3'-UTR mutant with a mutated seed region for predicted miR-4284 binding sites was constructed and named CXCL5MUT. The synthesized wild-type (CXCL5WT) or mutant (CXCL5MUT) sequence was cloned into the pmirGLO vector containing the firefly luciferase structural gene. For transfection, 5000 MSCs 
per well were seeded onto 96-well plates, and each construct was co-transfected with miR-4284 mimics or a negative control using Lipofectamine 3000 Transfection Reagent (Invitrogen). After $36 \mathrm{~h}$, cell lysates were harvested, and luciferase activity was determined using DualLuciferase $^{\circledast}$ Reporter (DLR ${ }^{\mathrm{Tw}}$ ) Assay System (Promega) according to the manufacturer's instructions. The ratio of firefly to Renilla luciferase activity was calculated as the relative luciferase activity.

\section{Statistical analysis}

Statistical analysis was performed using SPSS 20.0 software (Chicago, IL, USA). Comparisons between two groups were performed using Student's $t$ test. In addition, differences among three or more groups were analyzed by one-way analysis of variance. A value of $p<$ 0.05 was considered significant.

\section{Results}

\section{ASMSCs exhibit a stronger ability to inhibit} osteoclastogenesis than HDMSCs

To investigate differences in the ability of HDMSCs and ASMSCs to inhibit osteoclastogenesis, we cultured CD14 ${ }^{+}$ monocytes with MSCs; the medium was supplemented with M-CSF and RANKL to induce osteoclast differentiation. Purification of $\mathrm{CD}_{14} 4^{+}$monocytes was $>95 \%$, as confirmed by flow cytometry (Supplementary Figure S1). TRAP staining assays were performed on days 6,9 , and 12 , and the number of TRAP ${ }^{+}$osteoclasts was significantly decreased after co-culture with MSCs, with fewer osteoclasts in the ASMSC group than that of HDMSC group (Fig. 1a). The TRAP staining assay quantification is shown in Fig. 1b. As the band of F-actin containing podosomes in osteoclasts participates in bone resorption, the F-actin assays indicated that these osteoclast-like cells can resorb bone (Fig. 1c). Furthermore, pit formation was decreased after culture with MSCs (Fig. 1d). Most notably, CD14 ${ }^{+}$monocytes cultured with ASMSCs exhibit difficulty in differentiating into osteoclasts and causing decreased bone resorption compared with cells cultured with HDMSCs (Fig. 1d). The bone resorption assay results are summarized in Fig. 1e. These findings indicate that ASMSCs have a stronger capacity than HDMSCs to inhibit osteoclastogenesis.

As presented in Fig. 1f, qRT-PCR revealed that osteoclasts cultured with ASMSCs expressed lower levels of TRAP, CTSK, and NFATc1 than cells cultured with HDMSCs, and the results of the western blot analyses were consistent (Fig. 1g, h). Moreover, osteoclasts cultured with ASMSCs expressed lower levels of phosphorylated p65 than osteoclasts cultured with HDMSCs, though no differences in JNK, p38, AKT, and ERK pathway components were found (Fig. 1i, j). These results suggest that the p65 pathway is essential for the ASMSCmediated inhibition of osteoclastogenesis.

\section{ASMSCs secrete more CXCL5 than do HDMSCs}

To identify factors involved in the excessive inhibition of osteoclastogenesis by ASMSCs, we analyzed culture supernatants using a Human XL Cytokine Array Kit. Several cytokines with abnormal expression were found in the culture supernatants of ASMSCs, including CXCL5, angiogenin (ANG), GRO $\alpha$ and thrombospondin1 (THBS1) (Fig. 2a, b). qRT-PCR assays were performed to confirm expression levels of these cytokines in ASMSCs and HDMSCs. Of note, the most prominent increase was found for CXCL5 (Fig. 2c), and the results of western blot assays were consistent with these qRT-PCR results (Fig. 2d, e). Increased release of CXCL5 from ASMSCs was also confirmed by ELISA (Fig. 2f).

\section{Effect of sh-CXCL5 on the inhibition of osteoclastogenesis by MSCs}

To determine whether CXCL5 is the key cytokine for the observed increase in osteoclastogenesis inhibition by ASMSCs, we constructed a lentivirus encoding a short hairpin RNA targeting CXCL5 (sh-CXCL5). Both HDMSCs and ASMSCs were effectively transfected with the lentivirus (Supplementary Figure S2), and production of CXCL5 was reduced by at least $80 \%$ (Fig. 3a). After CXCL5 knockdown, the number of osteoclasts increased, with no notable difference in the number of osteoclasts between HDMSCs and ASMSCs (Fig. 3b, c). The results of $\mathrm{F}$-actin and bone resorption assays were consistent with the results of TRAP staining (Fig. $3 \mathrm{~d}-\mathrm{f}$ ), indicating that elevated CXCL5 was the main cause of the increased inhibition of osteoclastogenesis by ASMSCs.

\section{Exogenous CXCL5 inhibits osteoclastogenesis in a dose- dependent manner through the p65 pathway}

To investigate the effect of CXCL5 on osteoclastogenesis, we cultured $\mathrm{CD} 14^{+}$monocytes under osteoclastogenic conditions in the continuous presence of CXCL5 at different concentrations $(0,1,5,25,125 \mathrm{ng} / \mathrm{mL})$. As determined by TRAP staining, the number of TRAP ${ }^{+}$ osteoclasts gradually decreased as the concentration of CXCL5 increased (Fig. 4a, b). F-actin and bone resorption assays showed that bone resorption capacity also decreased with increasing concentration of CXCL5 (Fig. 4a, c). These data demonstrate that CXCL5 inhibits osteoclastogenesis in a dose-dependent manner.

qRT-PCR and western blot analyses showed that the expression levels of TRAP, CTSK, and NFATc1 in osteoclasts were decreased after stimulation with CXCL5 (Fig. 4d, f). In addition, phosphorylation of p65 was markedly decreased after stimulation with CXCL5, though the JNK, p38, AKT, and ERK pathways were not affected (Fig. 4g, h). These results show that CXCL5 inhibits osteoclastogenesis through the p65 pathway. 


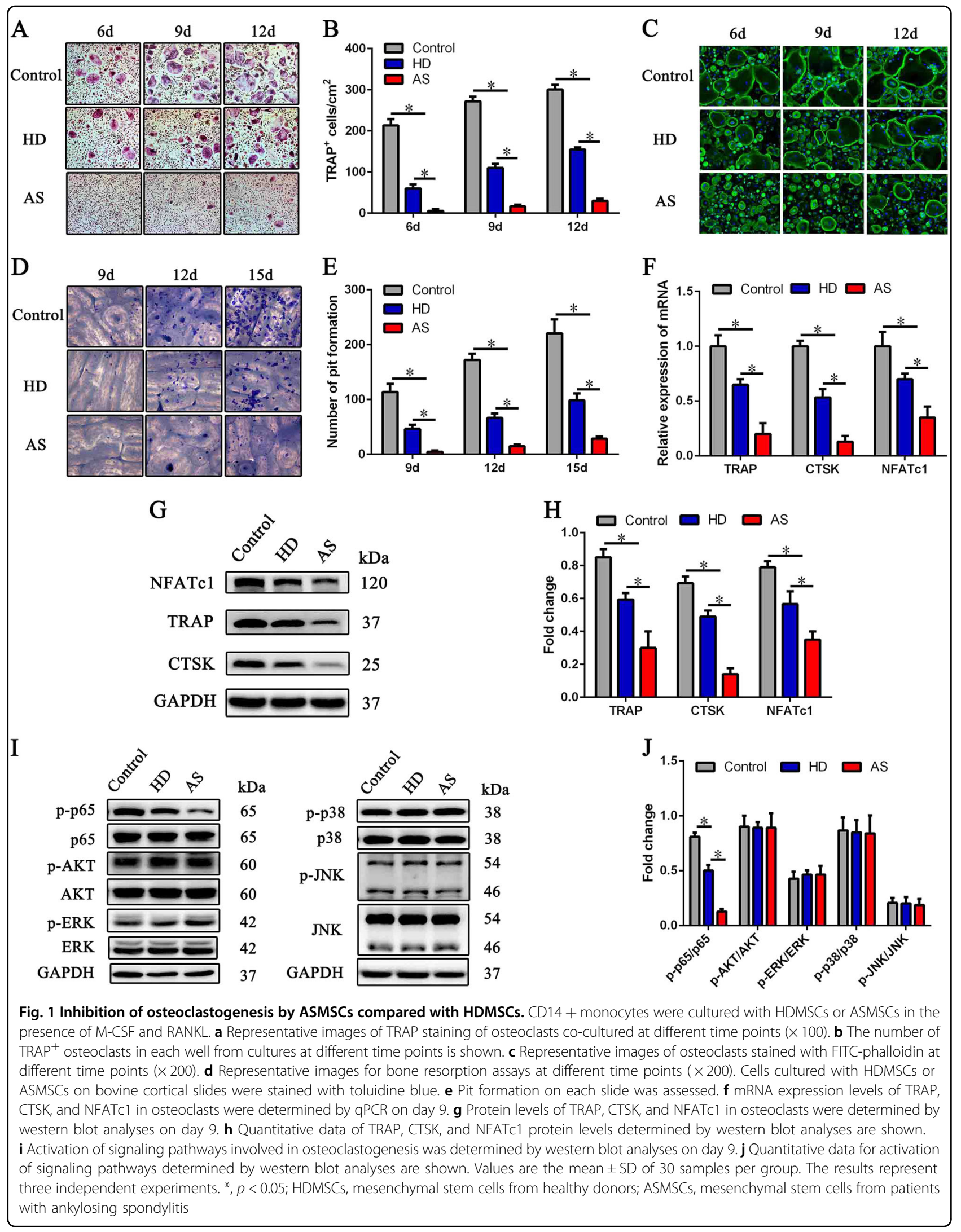



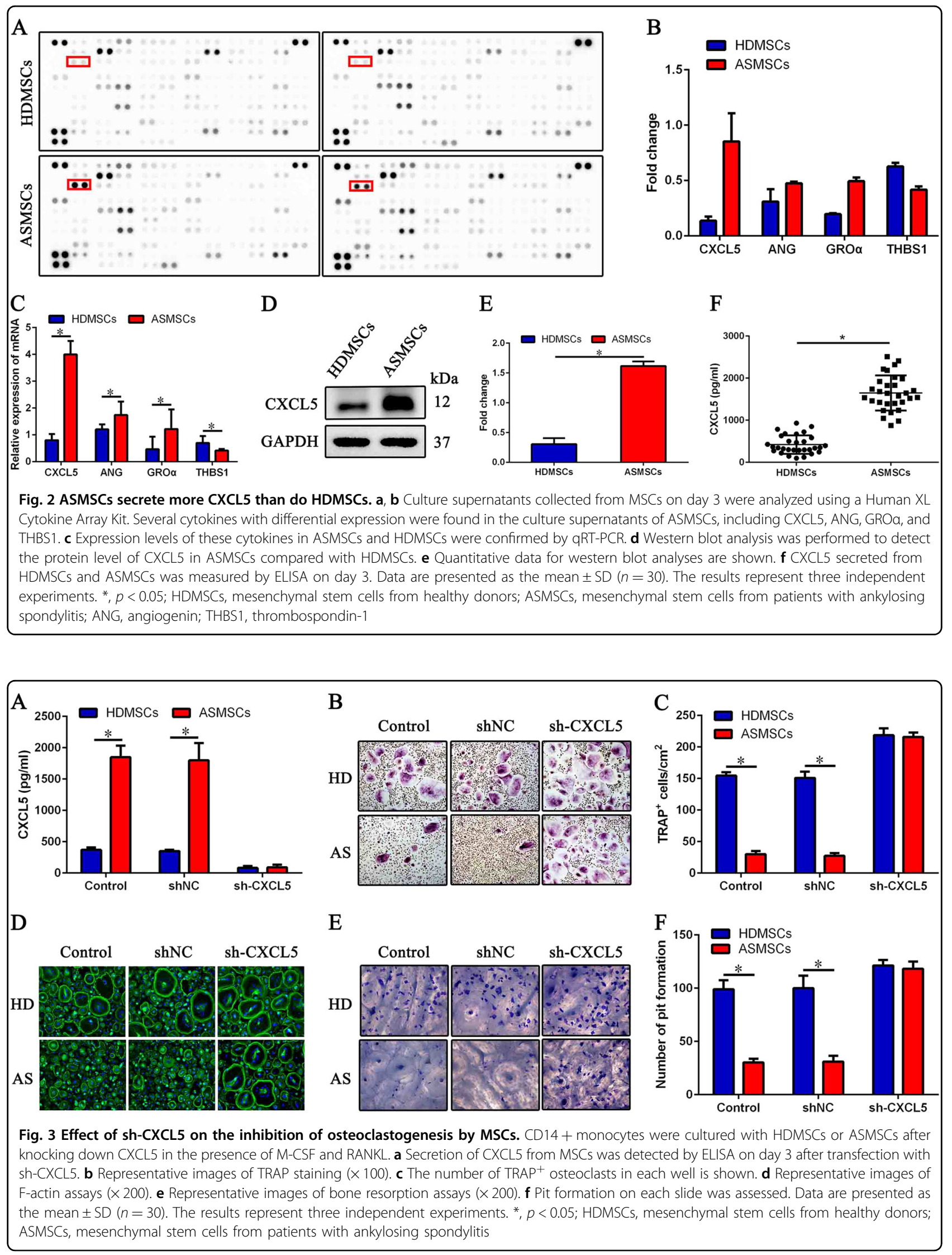


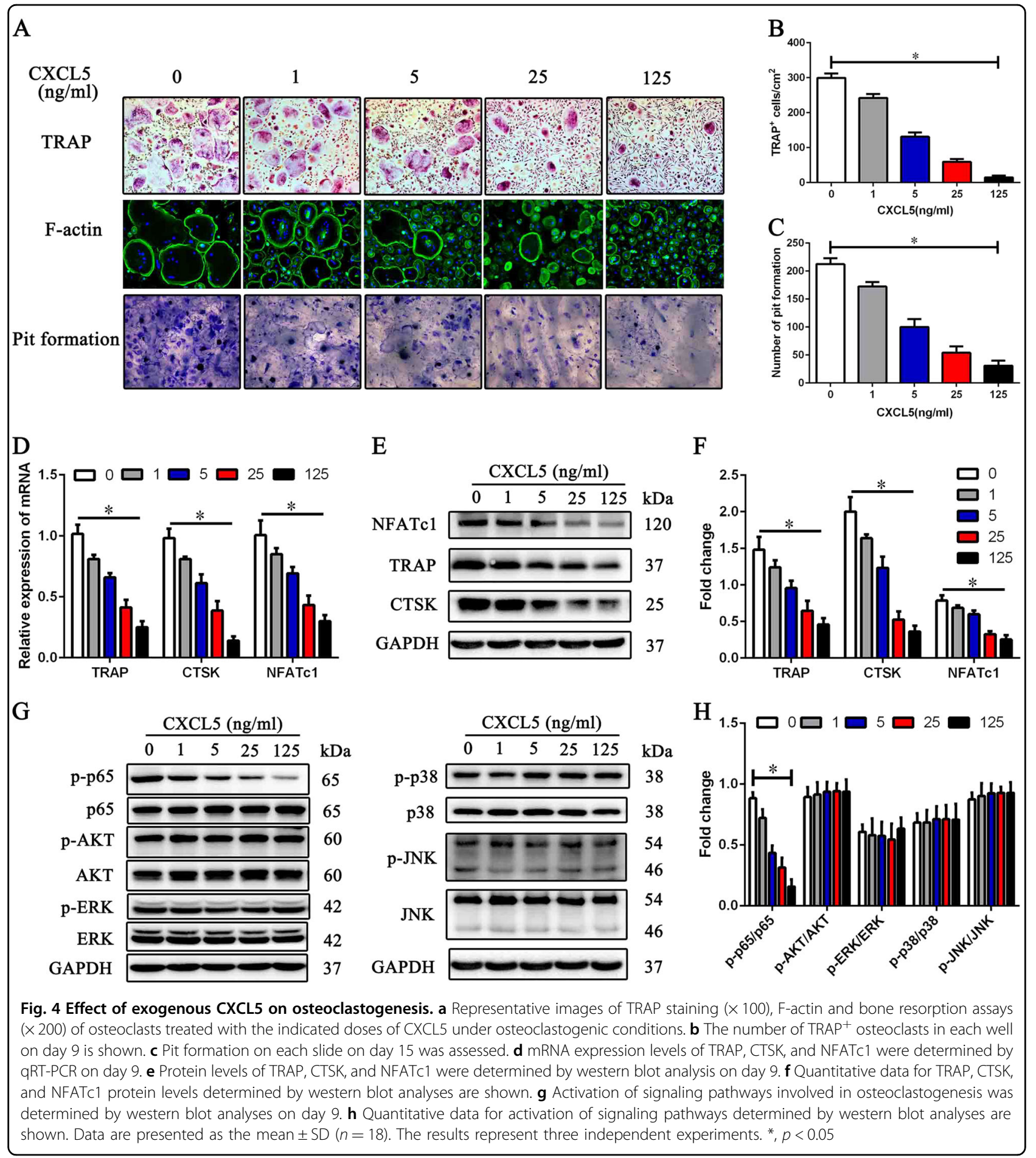

miR-4284 is decreased in ASMSCs and regulates expression of CXCL5

miRNAs can regulate the paracrine effects of MSCs and also participate in the pathogenesis of AS. To further explore the upstream molecular mechanism of upregulated CXCL5 expression in ASMSCs, we selected possible
miRNAs targeting CXCL5 using bioinformatics analysis of three databases (TargetScan, miRBase, and miRDB). Seven miRNAs had the highest scores for CXCL5 targeting (Fig. 5a), and qRT-PCR showed only miR-4284 to be downregulated in ASMSCs (Fig. 5b). To investigate whether miR-4284 regulates expression of CXCL5, we 


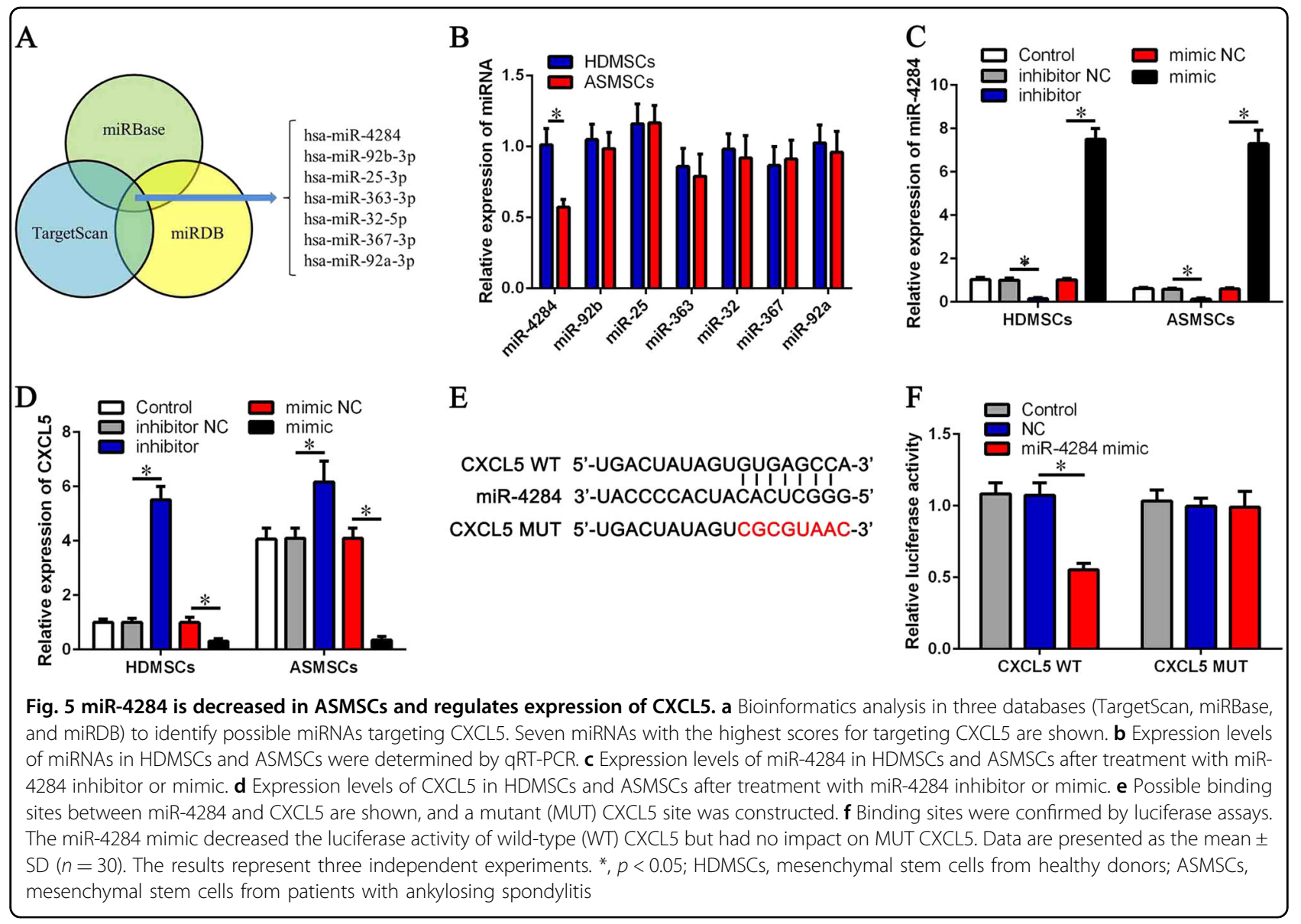

transfected MSCs with a miR-4284 inhibitor or mimic and found that CXCL5 was upregulated in the miR-4284 inhibitor group and downregulated in the miR-4284 mimic group (Fig. 5c, d). Moreover, there was no difference in CXCL5 expression between HDMSCs and ASMSCs transfected with miR-4284 inhibitor or mimic (Fig. 5c, d). We next performed luciferase reporter assays to further verify the direct interaction between miR-4284 and CXCL5 and predicted binding sites in the $3^{\prime}$-UTR of CXCL5. Using WT and MUT CXCL5 (Fig. 5e) constructs, luciferase reporter assays revealed significantly decreased luciferase activity for WT CXCL5 owing to the miR-4284 mimic, with no impact on MUT CXCL5 (Fig. 5f). These results demonstrate that miR-4284 is downregulated in ASMSCs, inducing elevated CXCL5 expression, which may influence osteoclastogenesis.

\section{miR-4284 can regulate MSC-mediated inhibition of osteoclastogenesis}

To explore the effect of miR-4284 on osteoclastogenesis, we transfected MSCs with the miR-4284 inhibitor or mimic and found expression of miR-4284 to be reduced by at least $80 \%$ (Fig. 6a). The number of osteoclasts cultured with HDMSCs was also decreased, but there was no difference compared with that of cells cultured with ASMSCs (Fig. 6b, c). In addition, no difference in bone resorption capacity after transfection with the miR-4284 inhibitor was observed (Fig. 6d-f). Furthermore, expression of miR-4284 in MSCs was significantly increased after miR-4284 mimic transfection (Fig. 6g), and the number of osteoclasts cultured with ASMSCs recovered to the level of those cultured with HDMSCs (Fig. 6h, i). F-actin and bone resorption assays confirmed the results of TRAP staining (Fig. 6j-l). These results indicate that miR-4284 can reverse the effect of CXCL5 on the inhibition of osteoclastogenesis by MSCs.

\section{Discussion}

In this study, we found that the capacity to inhibit osteoclastogenesis was increased in ASMSCs compared with HDMSCs. Further analyses demonstrated CXCL5 to be the main cause of the abnormal inhibition of osteoclastogenesis by ASMSCs. Bioinformatics analysis predicted that miR-4284 interacts with CXCL5, and we found that miR-4284 was downregulated in ASMSCs. miR-4284 was also shown to regulate expression of CXCL5. Indeed, there were no differences in osteoclastogenesis inhibition between HDMSCs and ASMSCs after the addition of the 


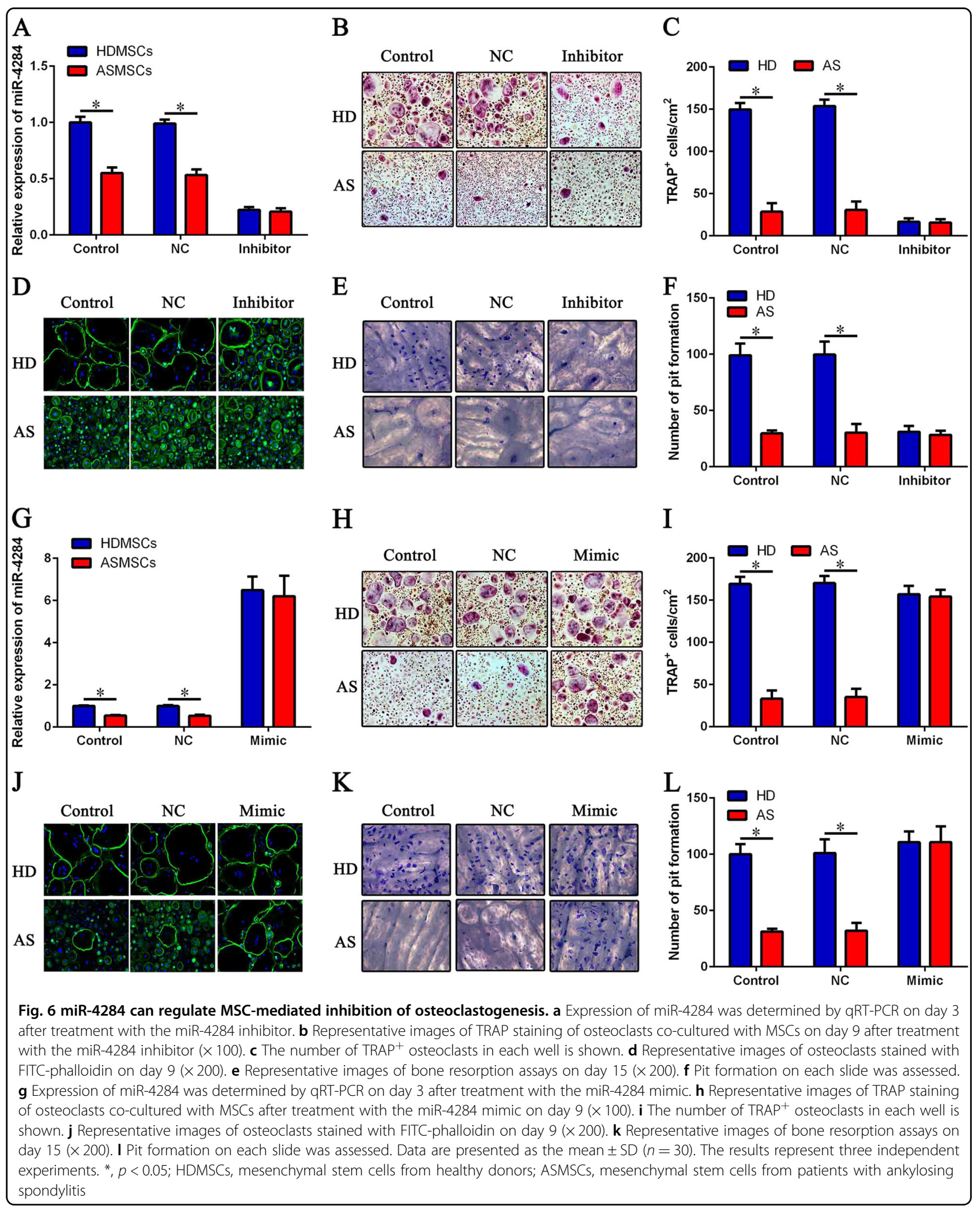

miR-4284 inhibitor or mimic. These data indicate that ASMSCs exhibit abnormal inhibition of osteoclastogenesis through the miR-4284/CXCL5 axis and that ASMSCs have a pathogenic role in AS by acting as a mediator of osteoclastogenesis inhibition, which may cause pathological osteogenesis in AS. 
Bone remodeling in vivo depends on the balance between osteoblasts, which are derived from MSCs, and osteoclasts, which are derived from monocytes ${ }^{8-10}$. Either dysfunction or abnormal mutual regulation of these two cells can lead to bone homeostasis disorders, which contribute to abnormal osteogenesis in rheumatic disease $\mathrm{e}^{28}$. Pathological osteogenesis is one of the central characteristics of $\mathrm{AS}^{4}$. Previously, we demonstrated that ASMSCs outperformed HDMSCs in osteogenic differentiation, one of the most important mechanisms of pathological osteogenesis in $\mathrm{AS}^{7}$. In addition, a recent study reported that the osteoclast differentiation ability of monocytes from AS patients is equal to that of HD monocytes when cultured in vitro ${ }^{29}$. Nonetheless, the mutual regulation of these two cells in AS has remained unclear. In this study, we found that the capacity of ASMSCs to inhibit osteoclastogenesis was stronger than that of HDMSCs. During osteogenic differentiation of MSCs, the capacity of MSCs to inhibit osteoclastogenesis was not significantly changed (Supplement Figure S3). From another point of view, these results highlight the importance of ASMSCs in the pathogenesis of AS, not only through their higher osteogenic differentiation capability but also through their stronger inhibition of osteoclastogenesis.

Many studies have shown that MSCs can regulate the differentiation of osteoclasts, yet their effect on osteoclastogenesis is controversial. In our study, both HDMSCs and ASMSCs were able to inhibit osteoclastogenesis. Early studies suggest that MSCs promote osteoclastogenesis $^{30,31}$, whereas recent studies demonstrate that MSCs inhibit osteoclastogenesis ${ }^{11,12}$. We identified several differences among these studies. First, the types of cells cultured with MSCs differed among studies. For example, most studies showing that MSCs inhibit osteoclastogenesis, consistent with our results, utilized $\mathrm{PBMCs}^{11,12}$, whereas studies with opposing results used CD34 ${ }^{+}$ hematopoietic stem cells or RAW264.7 cells in their experiments $^{30,31}$. Second, treatment of MSCs with various stimuli has different effects on osteoclastogenesis. For example, pressure-loaded MSCs promote osteoclastogenesis, but MSCs under no pressure have the opposite effect $^{31}$. Finally, culture conditions were also different. Some studies reporting results consistent with ours added RANKL to the medium ${ }^{11,12}$, though others did not $^{30,31}$. Overall, the above factors may account for the different effects of MSCs on osteoclastogenesis. Because all the cells used in our study were isolated from humans, our results agrees with human physiological and pathological processes, and this approach may be more suitable for the research of human diseases than other types of studies. Regardless, the effect of MSCs on osteoclasts needs to be further confirmed in vivo.

To investigate the mechanism underlying the stronger inhibition of osteoclastogenesis by ASMSCs, we performed cytokine array assays to identify possible cytokines related to this process and found CXCL5 to be significantly elevated in ASMSCs, causing abnormal inhibition of osteoclastogenesis. However, mechanism responsible for MSC-mediated inhibition of osteoclastogenesis has not been fully elucidated. Oshita $\mathrm{K}^{11}$ found that MSCs inhibit osteoclastogenesis through osteoprotegerin production, and Audrey Varin reported that the inhibitory effect of MSCs on osteoclast formation depends on CD200 expression $^{12}$. In our study, oversecretion of CXCL5 was the main reason for the abnormal inhibition of osteoclastogenesis by ASMSCs. These data suggest that MSCs inhibit osteoclastogenesis through a variety of cytokines instead of a single mediator. Our results, together with previous findings, may help elucidate the mechanism by which MSCs inhibit osteoclastogenesis. Previous studies have shown that CXCL5 is involved in a variety of inflammatory diseases, such as RA and pediatric ulcerative colitis ${ }^{20,21}$, though the effect of CXCL5 on AS is unknown. In particular, our research is the first to show that CXCL5 is elevated in AS and inhibits osteoclastogenesis. Our findings reveal a new function for CXCL5, and more roles of CXCL5 in AS should be explored in the future.

miRNAs expressed in MSCs have been shown to regulate differentiation as well as proliferation, senescence, migration, and survival ${ }^{32-35}$. Recent studies have also focused on the function of miRNAs in the paracrine effects of $\mathrm{MSCs}^{34}$. These miRNAs may modulate expression of proteins secreted from MSCs. Our study demonstrated that miR-4284 is downregulated in ASMSCs, promoting CXCL5 secretion from ASMSCs and inhibiting osteoclastogenesis. One recent study found that miR-4284 can regulate expression of CXCL5, but the researchers did not construct a CXCL5 3'-UTR mutant with a mutated seed region for the predicted miR-4284binding sites to verify these $\operatorname{sites}^{36}$. Our results were consistent with their findings, and we also constructed such a CXCL5 3'-UTR mutant to confirm miR-4284 binding sites. Many studies have found that miRNAs are associated with inflammation in $\mathrm{AS}^{37,38}$. miR-130a is downregulated in PBMCs from AS patients, inducing inflammation by enhancing TNF-1 $\alpha$ expression ${ }^{39}$. In $\mathrm{T}$ cells from AS patients, miRNA-199a-5p regulates autophagy and proinflammatory cytokine production by targeting RHEB $^{40}$. However, research on the role of miRNAs in the pathologic osteogenesis of AS is limited to date. Our results indicate that miRNAs may play an important role in the pathologic osteogenesis of AS, and further studies are needed to determine the unique miRNA signatures in AS pathologic osteogenesis.

In conclusion, ASMSCs appear to have a stronger ability to inhibit osteoclastogenesis than HDMSCs via suppression of the p65 pathway, which is regulated by the miR4284/CXCL5 axis. These results may help illuminate the 
mechanism of pathologic osteogenesis and provide new ideas for AS treatments. Nonetheless, our research also has some limitations, such as the lack of an animal model to study the function of ASMSCs in vivo. In addition, the reason why miR-4284 is downregulated in ASMSCs remains elusive. Further studies are needed to address these issues.

\section{Acknowledgements}

This study was financially supported by the Industrial Technology Research and Development Funding Project of Guangdong Province (20160911), the National Natural Science Foundation of China $(81672128,81702120)$, and the Science and Technology Plan of Guangzhou City (201704020045).

\section{Conflict of interest}

The authors declare that they have no conflict of interest.

\section{Publisher's note}

Springer Nature remains neutral with regard to jurisdictional claims in published maps and institutional affliations.

Supplementary Information accompanies this paper at (https://doi.org/ 10.1038/s41419-019-1448-x).

Received: 14 November 2018 Revised: 24 January 2019 Accepted: 12 February 2019

Published online: 25 February 2019

\section{References}

1. Sieper, J. \& Poddubnyy, D. Axial spondyloarthritis. Lancet 390, 73-84 (2017).

2. DeLay, M. L. et al. HLA-B27 misfolding and the unfolded protein response augment interleukin-23 production and are associated with Th17 activation in transgenic rats. Arthritis Rheum. 60, 2633-2643 (2009).

3. Smith, J. A. Update on ankylosing spondylitis: current concepts in pathogenesis. Curr. Allergy Asthma Rep. 15, 489 (2015).

4. Braun, J. \& Sieper, J. Ankylosing spondylitis. Lancet 369, 1379-1390 (2007).

5. Taurog, J. D., Chhabra, A. \& Colbert, R. A. Ankylosing spondylitis and axial spondyloarthritis. N. Engl. J. Med. 374, 2563-2574 (2016).

6. Yue, R., Zhou, B. O., Shimada, I. S., Zhao, Z. \& Morrison, S. J. Leptin receptor promotes adipogenesis and reduces osteogenesis by regulating mesenchymal stromal cells in adult bone marrow. Cell Stem Cell 18, 782-796 (2016).

7. Xie, Z. et al. Imbalance between bone morphogenetic protein 2 and noggin induces abnormal osteogenic differentiation of mesenchymal stem cells in ankylosing spondylitis. Arthritis Rheum. 68, 430-440 (2016).

8. Crane, J. L. \& Cao, X. Bone marrow mesenchymal stem cells and TGF-beta signaling in bone remodeling. J. Clin. Invest. 124, 466-472 (2014).

9. Feng, X. \& McDonald, J. M. Disorders of bone remodeling. Annu. Rev. Pathol. 6 , 121-145 (2011).

10. Li, S. D., Zhai, Q. L. \& Qiu, L. G. Imbalance between bone formation and resorption in hematopoietic stem/progenitor cells mobilization. Transplant. Proc. 43, 3920-3926 (2011).

11. Oshita, K. et al. Human mesenchymal stem cells inhibit osteoclastogenesis through osteoprotegerin production. Arthritis Rheum. 63, 1658-1667 (2011).

12. Varin, A., Pontikoglou, C., Labat, E., Deschaseaux, F. \& Sensebe, L. CD200R/ CD200 inhibits osteoclastogenesis: new mechanism of osteoclast control by mesenchymal stem cells in human. PLOS ONE 8, e72831 (2013).

13. Massey, H. M. \& Flanagan, A. M. Human osteoclasts derive from CD14-positive monocytes. Br. J. Haematol. 106, 167-170 (1999).

14. Okamoto, K. et al. Osteoimmunology: the conceptual framework unifying the immune and skeletal systems. Physiol. Rev. 97, 1295-1349 (2017).

15. Boyle, W. J., Simonet, W. S. \& Lacey, D. L. Osteoclast differentiation and activation. Nature 423, 337-342 (2003).
16. Takayanagi, H. Osteoimmunology and the effects of the immune system on bone. Nat. Rev. Rheumatol. 5, 667-676 (2009).

17. Kim, M. S. et al. MCP-1-induced human osteoclast-like cells are tartrate-resistant acid phosphatase, NFATC1, and calcitonin receptor-positive but require receptor activator of NFkappaB ligand for bone resorption. J. Biol. Chem. 281, 1274-1285 (2006).

18. Kim, H. R. et al. Reciprocal activation of CD4+T cells and synovial fibroblasts by stromal cell-derived factor 1 promotes RANKL expression and osteoclastogenesis in rheumatoid arthritis. Arthritis Rheum. 66, 538-548 (2014).

19. Mei, J. et al. CXCL5 regulates chemokine scavenging and pulmonary host defense to bacterial infection. Immunity 33, 106-117 (2010).

20. Yoshida, K. et al. Citrullination of epithelial neutrophil-activating peptide 78/ CXCL5 results in conversion from a non-monocyte-recruiting chemokine to a monocyte-recruiting chemokine. Arthritis Rheum. 66, 2716-2727 (2014).

21. Nouailles, G. et al. CXCL5-secreting pulmonary epithelial cells drive destructive neutrophilic inflammation in tuberculosis. J. Clin. Invest. 124, 1268-1282 (2014).

22. Li, Z., Wong, S. H., Shen, J., Chan, M. T. \& Wu, W. K. The role of MicroRNAS in ankylosing spondylitis. Medicine (Baltim.) 95, e3325 (2016).

23. Wittmann, J. \& Jack, H. M. microRNAs in rheumatoid arthritis: midget RNAs with a giant impact. Ann. Rheum. Dis. 70, i92-i96 (2011).

24. Perez-Sanchez, C. et al. Circulating microRNAs as potential biomarkers of disease activity and structural damage in ankylosing spondylitis patients. Hum. Mol. Genet. 27, 875-890 (2018).

25. van der Linden, S., Valkenburg, H. A. \& Cats, A. Evaluation of diagnostic criteria for ankylosing spondylitis. A proposal for modification of the New York criteria. Arthritis Rheum. 27, 361-368 (1984).

26. Xie, Z. et al. MCP1 triggers monocyte dysfunctions during abnormal osteogenic differentiation of mesenchymal stem cells in ankylosing spondylitis. J. Mol. Med. (Berl.) 95, 143-154 (2017)

27. $L i, Y$. et al. Whole genome expression profiling and signal pathway screening of MSCs in ankylosing spondylitis. Stem Cells Int. 2014, 913050 (2014).

28. Walsh, N. C. \& Gravallese, E. M. Bone remodeling in rheumatic disease: a question of balance. Immunol. Rev. 233, 301-312 (2010).

29. Perpetuo, I. P. et al. Ankylosing Spondylitis patients have impaired osteoclast gene expression in circulating osteoclast precursors. Front. Med. (Lausanne) $\mathbf{4}$ 5 (2017).

30. Mbalaviele, G. et al. Human mesenchymal stem cells promote human osteoclast differentiation from CD34+bone marrow hematopoietic progenitors. Endocrinology 140, 3736-3743 (1999).

31. Liu, J. et al. Pressure-loaded MSCs during early osteodifferentiation promote osteoclastogenesis by increase of RANKL/OPG ratio. Ann. Biomed. Eng. 37, 794-802 (2009)

32. Vicente, R., Noel, D., Pers, Y. M., Apparailly, F. \& Jorgensen, C. Deregulation and therapeutic potential of microRNAs in arthritic diseases. Nat. Rev. Rheumatol. 12, 211-220 (2016).

33. Lakshmipathy, U. \& Hart, R. P. Concise review: MicroRNA expression in multipotent mesenchymal stromal cells. Stem Cells 26, 356-363 (2008).

34. Clark, E. A.r Kalomoiris, S., Nolta, J. A. \& Fierro, F. A. Concise review: microrna function in multipotent mesenchymal stromal cells. Stem Cells 32, 1074-1082 (2014).

35. Chen, Q. et al. Fate decision of mesenchymal stem cells: adipocytes or osteoblasts? Cell Death Differ. 23, 1128-1139 (2016).

36. Koukos, G. et al. A microRNA signature in pediatric ulcerative colitis: deregulation of the miR-4284/CXCL5 pathway in the intestinal epithelium. Inflamm. Bowel Dis. 21, 996-1005 (2015)

37. Mohammadi, $\mathrm{H}$. et al. MicroRNA implications in the etiopathogenesis of ankylosing spondylitis. J. Cell Physiol. 233, 5564-5573 (2018).

38. Lai, N. S. et al. Aberrant expression of microRNAs in T cells from patients with ankylosing spondylitis contributes to the immunopathogenesis. Clin. Exp. Immunol. 173, 47-57 (2013).

39. Jiang, Y. \& Wang, L. Role of histone deacetylase 3 in ankylosing spondylitis via negative feedback loop with microRNA-130a and enhancement of tumor necrosis factor-1alpha expression in peripheral blood mononuclear cells. Mol. Med. Rep. 13, 35-40 (2016).

40. Wang, Y., Luo, J., Wang, X., Yang, B. \& Cui, L. MicroRNA-199a-5p induced autophagy and inhibits the pathogenesis of ankylosing spondylitis by modulating the mTOR signaling via directly targeting ras homolog enriched in Brain (Rheb). Cell Physiol. Biochem. 42, 2481-2491 (2017). 